\title{
Effectiveness of combined suprascapular nerve and costoclavicular brachial plexus block for arthroscopic shoulder surgery: a prospective observational study
}

\section{Byung-Gun Kim}

Inha University College of Medicine

\section{Woojoo Lee}

Inha University

Hyunzu Kim

Inha University College of Medicine

Chunwoo Yang ( $\square$ everycw@daum.net)

Inha University College of Medicine https://orcid.org/0000-0002-9147-3879

\section{Yoon Sang Jeon}

Inha University College of Medicine

Nayoung Tae

Inha University College of Medicine

Hongseok Kim

Inha University College of Medicine

Research article

Keywords: arthroscopic shoulder surgery, brachial plexus, nerve block, suprascapular nerve

Posted Date: September 8th, 2019

DOI: https://doi.org/10.21203/rs.2.14086/v1

License: (c) (1) This work is licensed under a Creative Commons Attribution 4.0 International License.

Read Full License 


\section{Abstract}

Background Although interscalene brachial plexus block provides effective anesthesia and analgesia for shoulder surgery, one major drawback of interscalene brachial plexus block is ipsilateral phrenic nerve palsy. Several diaphragm-sparing nerve blocks have been suggested. We prospectively evaluated the effectiveness of combined suprascapular nerve and costoclavicular brachial plexus block for postoperative analgesia following arthroscopic shoulder surgery.

Methods Nineteen patients scheduled for arthroscopic shoulder surgery received combined ultrasoundguided suprascapular nerve and costoclavicular brachial plexus block. Pain scores, use of supplemental analgesia, incidence of phrenic nerve palsy, block-related outcomes, patient satisfaction, and adverse effects were assessed.

Results The block was successful in 17 patients (89.5\%). Postoperative pain control was effective during the first $24 \mathrm{~h}$ after surgery. Phrenic nerve palsy did not occur ( $0 \%, 95 \%$ confidential interval $0-17 \%)$. There were no severe adverse events. Patients reported a high degree of satisfaction.

Conclusion Combined suprascapular nerve and costoclavicular brachial plexus block provided effective postoperative analgesia for arthroscopic shoulder surgery without causing phrenic nerve palsy.

\section{Background}

Despite its efficacy for shoulder surgery, one major drawback of interscalene brachial plexus block (ISB) is ipsilateral phrenic nerve palsy, which is a concern in patients with respiratory disease or obesity [1].

Several diaphragm-sparing nerve blocks such as $\mathrm{C} 7$ nerve root block,[2] supraclavicular brachial plexus block [3, 4], and distal nerve blocks have been investigated [5, 6]. While supraclavicular brachial plexus block provided similar analgesia compared to ISB for shoulder surgery [3, 4], most distal nerve blocks are less effective compared to ISB, especially immediate postoperative period [5-7]. An alternative to ISB is combined proximal suprascapular nerve block (SSNB) and costoclavicular brachial plexus block (CCB). Theoretically, since these blocks target the relevant nerves more proximally, there is a possibility that their combination will result in more complete anesthetic coverage of the shoulder joint. However, this has not been studied.

Therefore, we aimed to evaluate the clinical efficacy of combined SSNB-CCB in arthroscopic shoulder surgery. We hypothesized that combined SSNB-CCB provides effective postoperative analgesia for arthroscopic shoulder surgery without causing phrenic nerve palsy.

\section{Methods}

This prospective, observational study was approved by the institutional review board, and registered before patient enrollment (the Clinical Trial Registry of Korea: https://cris.nih.go.kr/cris/index.jsp, 
Identifier: KCT0003125, Principal investigator: Chunwoo Yang, Date of registration: 8.22.2018). Adult inpatients (20-80 years of age, American Society of Anesthesiologists physical status 1-3) scheduled for arthroscopic shoulder surgery were considered eligible for inclusion. Patients with severe respiratory disease, contraindication to regional anesthesia, body mass index $>35 \mathrm{~kg} / \mathrm{m}^{2}$, pre-existing neuropathy, allergy to study medications, or pregnancy were excluded. All patients provided written informed consent and were fully informed of the risks and benefits of participating, including the risk of block failure and the potential need to repeat the procedure.

ASA monitoring was applied along with supplemental oxygen upon arrival to the induction room and utilized throughout the procedure. All blocks were performed by the same anesthetist. We used an ultrasound (US) system (Vivid q, GE Healthcare, WI, USA) with a high frequency linear probe (6-13 MHz) for guidance. Blocks were performed with a $50 \mathrm{~mm}$ insulated needle (UniPlex NanoLine, Pajunk, Geisingen, Germany) using a nerve stimulator (MultiStim, Pajunk) for localization. All patients received intravenous (IV) midazolam (1-3 mg) and fentanyl $(25-50 \mu \mathrm{g})$ for sedation and anxiolysis before the block.

\section{Block technique}

The CCB was performed first using a previously described technique [8]. Patients were positioned supine with the head turned to the contralateral side. The US probe was initially placed below the clavicle and over the medial infraclavicular fossa. Subsequently, the probe was tilted slightly cephalad to visualize the costoclavicular space and the three cords of the brachial plexus. After skin infiltration, the needle was advanced under US guidance in-plane and from a lateral-to-medial direction until its tip was located in the middle of all three cords. After negative aspiration, $12 \mathrm{ml}$ ropivacaine $0.5 \%$ was slowly injected.

Proximal SSNB was performed using previously described techniques [9]. Patients were positioned supine with the head turned to the contralateral side. Under sterile conditions, the US probe was placed in the lateral side of the neck at the level of the cricoid cartilage. The suprascapular nerve was traced distally from the superior trunk or $\mathrm{C} 5$ nerve root to select a puncture site as far lateral as possible to avoid local anesthetic (LA) spread to the supraclavicular brachial plexus. This nerve is usually located underneath the omohyoid muscle at the supraclavicular fossa. After skin infiltration, the needle was advanced in a lateral-to-medial direction using an in-plane technique until the tip was positioned at the suprascapular nerve. After confirming sustained muscle contraction with the nerve stimulator $(>0.2 \mathrm{~mA})$, $8 \mathrm{ml}$ ropivacaine $0.5 \%$ was slowly injected after negative aspiration. If the nerve was not visualized or stimulation did not elicit an appropriate motor response, LA was injected underneath the omohyoid muscle.

\section{Preoperative evaluation}


During block placement, block performance time, number of needle passes, patient discomfort, and any adverse events (such as vascular puncture, LA toxicity, and unintentional paresthesia) were recorded by the anesthesia nurse assisting the anesthetist performing the block. Block performance time was defined as the time from first block needle insertion to the end of the second block LA injection. The initial needle insertion was considered as the first pass. Any subsequent needle advancement that was preceded by a retraction of at least $10 \mathrm{~mm}$ counted as an additional pass. Patient discomfort during block placement was assessed using a numerical rating scale (NRS) with range $0-10(0$, no pain; 10 , worst imaginable pain).

Motor block (shoulder abduction for axillary nerve and shoulder external rotation for suprascapular nerve) was clinically assessed 30 min after block placement with a three-point scale (0, no block; 1 , paresis; and 2 , paralysis). Since the suprascapular and subscapular nerve typically do not contain cutaneous afferent fibers [10], sensory block of all nerves related to the shoulder joint was not tested. A successful block was defined as a patient discomfort rating of 0 in the post-anesthesia care unit (PACU) at 30 min with a block duration of more than $6 \mathrm{hr}$ postoperatively. Block duration was defined as the time interval between the end of the LA injection and the first pain sensation.

Diaphragmatic excursion was assessed before and $30 \mathrm{~min}$ after nerve blocks. With the patient in a supine position, a 4-MHz curvilinear US probe was used to perform the scan from a low intercostal or subcostal approach, using the liver on the right or spleen on the left as an acoustic window. Diaphragmatic excursion was measured during deep inspiration using M-mode ultrasonography. Complete, partial, and no diaphragmatic paralysis were individually defined as decrease between $75 \%$ and $100 \%$ (including the occurrence of paradoxical movement), decrease between $25 \%$ and $75 \%$, and a decrease of $<25 \%$ in diaphragmatic excursion, respectively.

\section{Perioperative management}

All patients received general anesthesia using IV propofol $(1-2 \mu \mathrm{g} / \mathrm{kg})$ with endotracheal intubation facilitated by IV cisatracurium $(0.1-0.2 \mu \mathrm{g} / \mathrm{kg})$. Anesthesia was maintained using an air/oxygen mixture and $2-3 \%$ sevoflurane. IV fentanyl $(1-2 \mu \mathrm{g} / \mathrm{kg})$ was administered when blood pressure increased by > $20 \%$ over the preoperative blood pressure. At the conclusion of surgery, residual paralysis was antagonized with pyridostigmine and glycopyrrolate if required.

In the PACU, resting pain score at 30 min was assessed, and use of supplemental analgesia was recorded. Patients with block failure were offered the option of either rescue ISB or IV fentanyl for adequate pain control. Postoperatively, all patients received $1 \mathrm{~g}$ of IV paracetamol every $6 \mathrm{~h}$ and $30 \mathrm{mg}$ of IV ketorolac every $12 \mathrm{~h}$ for the first $24 \mathrm{~h}$ after surgery irrespective of pain status. For rescue analgesia, patients were instructed to request analgesics (meperidine $25 \mathrm{mg} \mathrm{IV}$ ) if their pain score was greater than 4. 


\section{Outcome measures}

The primary outcome was resting postoperative pain at $30 \mathrm{~min}$ in the PACU. Secondary outcomes included procedure-related outcomes, the occurrence of phrenic nerve palsy, motor block, pain score at 6 $\mathrm{h}$ and $24 \mathrm{~h}$ after surgery, block duration, use of supplemental analgesia in the PACU and surgical ward, patient satisfaction as assessed by a NRS (range $0-10 ; 0$, dissatisfied; 10 , very satisfied), and any adverse events (paresthesia, dyspnea, and handgrip weakness).

\section{Statistical analysis}

Since most distal nerve blocks for shoulder surgery usually result in inferior analgesia in the immediate postoperative period, the primary outcome of this study was the patient discomfort score in the PACU at 30 min. Assuming a noninferiority margin of 1.5 points and a standard deviation (SD) of 1.7 based on a previous study [4], the necessary sample size for $a=0.05$ and $80 \%$ power was calculated to be 16 patients. To allow for possible dropouts, we included 20 patients.

Descriptive statistics were used to summarize the data, which are presented as means (SD), medians (interquartile range), or numbers (percent) where appropriate. Statistical analyses were performed using SPSS 19.0 version software (IBM, Armonk, NY, USA).

\section{Results}

Of the 20 patients who were enrolled, one patient was excluded due to conversion to open surgery. Nineteen patients were included in the analyses. Patient demographic and surgical data are shown in Table 1. Block-related outcomes are shown in Table 2. No vascular puncture, paresthesia, or LA toxicity was observed during block placement. Thirty minutes after combined SSNB-CCB, complete motor block of the axillary and suprascapular nerves was present in 8/19 (42.1\%) patients and partial motor block in $11 / 19$ (57.9\%) patients. Mean pre-block diaphragmatic excursion was $4.4 \mathrm{~cm}$ (0.7). Phrenic nerve palsy did not occur in any patient $30 \mathrm{~min}$ after the block $(0 \%, 95 \% \mathrm{Cl} 0-17 \%)$.

The block was successful in 17 patients (89.5\%). Two patients (10.5\%) reported pain (NRS 6 and 3, respectively) in the PACU. Of those, only one received fentanyl $100 \mu \mathrm{g} \mathrm{IV}$, and did not require a rescue block. Complete and partial handgrip weakness in the PACU was present in 8 (42\%) and 11 patients (58\%), respectively.

The median pain score $30 \mathrm{~min}, 6 \mathrm{~h}$, and $24 \mathrm{~h}$ after surgery were $0(0-0), 0(0-0)$ and $3(2-5)$, respectively (Fig. 1). Median postoperative meperidine use was $25 \mathrm{mg} \mathrm{(0-50)}$ during the first $24 \mathrm{hr}$ after surgery. The median patient satisfaction rating was $10(9-10)$. No severe complications occurred.

\section{Discussion}


This study shows that combined SSNB-CCB provides effective postoperative analgesia during the first 24 hours following arthroscopic shoulder surgery without causing phrenic nerve palsy.

Several diaphragm-sparing nerve blocks have been previously suggested and evaluated $[1-5,8]$. While supraclavicular brachial plexus block provided similar analgesia compared to ISB for shoulder surgery [3, 4], most distal nerve blocks are less effective compared to ISB, especially in the immediate postoperative period [5-7]. In this study, however, combined SSNB-CCB provided adequate pain control not only in the immediate postoperative period, but also $24 \mathrm{~h}$ after surgery. This improved analgesia might be explained by the proximal SSNB. A recent study demonstrated that local anesthetic can spread to the upper trunk of brachial plexus and its branches during proximal SSNB [11]. Another contributing factor is CCB.

Compared with lateral sagittal infraclavicular block, CCB might provide better blockade of the posterior and lateral cords with a small volume of LA, as the cords of the brachial plexus are clustered lateral to the axillary artery at the costoclavicular space [8].

In this study, phrenic nerve palsy did not occur in any patient. This suggests that combined SSNB-CCB might be advantageous in patients with a high risk of respiratory complications. However, the small sample size of our study does not guarantee that phrenic nerve palsy will not occur following combined SSNB-CCB. In addition, combined blocks theoretically have a risk of causing phrenic nerve palsy [11-13].

In summary, combined SSNB-CCB can be an effective alternative to ISB for postoperative analgesia following arthroscopic shoulder surgery. Further prospective randomized investigations are warranted to confirm these results.

\section{Abbreviations}

CCB, costoclavicular brachial plexus block; ISB, interscalene brachial plexus block; IV, intravenous; LA, local anesthetic; NRS, numerical rating scale; PACU, post-anesthesia care unit; SSNB, suprascapular nerve block; US, ultrasound.

\section{Declarations}

\section{Ethics approval and consent to participate}

This study was approved by the Inha University Hospital Institutional Review Board (\#2018-05-014004). All enrolled patients provided written informed consent.

\section{Consent for publication}

Not applicable.

\section{Availability of data and materials}


The datasets used and/or analyzed during the current study are available from the corresponding author on reasonable request.

\section{Competing interests}

The authors have no conflicts of interest to disclose.

\section{Funding}

This work was supported by Inha University Research Grant (number 60536-01).

\section{Author' contributions}

BGK helped in conduct of the study, data analysis, and manuscript preparation. WL helped in study design, data analysis, and manuscript preparation. HK helped in data analysis and manuscript preparation. CY helped in study design, conduct of study, data analysis, and manuscript preparation. YSJ helped in study design and manuscript preparation. NT helped in conduct of the study, data collection, and manuscript preparation. HK helped in conduct of the study, data collection, and manuscript preparation. All authors read and approved the final manuscript.

\section{Acknowledgements}

None

\section{Author details}

${ }^{1}$ Department of Anesthesiology and Pain Medicine, Inha University College of Medicine, Incheon, South Korea. ${ }^{2}$ Department of Statistics, Inha University, Incheon, South Korea. ${ }^{3}$ Department of Orthopedic surgery, Inha University College of Medicine, Incheon, South Korea.

\section{References}

1. Melton MS, Monroe HE, Qi W et al. Effect of interscalene brachial plexus block on the pulmonary function of obese patients: a prospective, observational cohort study. Anesth Analg. 2017;125:3139.

2. Renes SH, Rettig HC, Gielen MJ, Wilder-Smith OH, van Geffen GJ. Ultrasound-guided low-dose interscalene brachial plexus block reduces the incidence of hemidiaphragmatic paresis. Reg Anesth Pain Med. 2009;34:498-502. 
3. Kim BG, Han JU, Song JH, et al. A comparison of ultrasound-guided interscalene and supraclavicular blocks for post-operative analgesia after shoulder surgery. Acta Anaesthesiol Scand. 2017;61:42735 .

4. Aliste J, Bravo D, Fernandez D, et al. A randomized comparison between interscalene and smallvolume supraclavicular blocks for arthroscopic shoulder surgery. Reg Anesth Pain Med. 2018;43:590-5.

5. Neuts A, Stessel B, Wouters PF, et al. Selective suprascapular and axillary nerve block versus interscalene plexus block for pain control after arthroscopic shoulder surgery: a noninferiority randomized parallel-controlled clinical trial. Reg Anesth Pain Med. 2018; 43:738-44.

6. Aliste J, Bravo D, Finlayson RJ, Tran DQ. A randomized comparison between interscalene and combined infraclavicular-suprascapular blocks for arthroscopic shoulder surgery. Can J Anesth. 2018;65:280-7.

7. Dhir S, Sondekoppam RV, Sharma R, Ganapathy S, Athwal GS. A comparison of combined suprascapular and axillary nerve blocks to interscalene nerve block for analgesia in arthroscopic shoulder surgery: an equivalence study. Reg Anesth Pain Med. 2016;41:564-71.

8. Li JW, Songthamwat B, Samy W, Sala-Blanch X, Karmakar MK. Ultrasound-guided costoclavicular brachial plexus block: sonoanatomy, technique, and block dynamics. Reg Anesth Pain Med. 2017;42:233-40.

9. Siegenthaler A, Moriggl B, Mlekusch S, et al. Ultrasound-guided suprascapular nerve block, description of a novel supraclavicular approach. Reg Anesth Pain Med. 2012;37:325-8.

10. Ajmani ML The cutaneous branch of the human suprascapular nerve. J Anat. 1994;185:439-42.

11. Laumonerie P, Ferre F, Cances J, et al. Ultrasound-guided proximal suprascapular nerve block: a cadaveric study. Clin Anat. 2018;31:824-9.

12. Rettig HC, Gielen MJ, Boersma E, Klein J, Groen GJ. Vertical infraclavicular block of the brachial plexus: effects on hemidiaphragmatic movement and ventilatory function. Reg Anesth Pain Med. 2005;30:529-35.

13. Petrar SD, Seltenrich ME, Head SJ, Schwarz SK. Hemidiaphragmatic paralysis following ultrasoundguided supraclavicular versus infraclavicular brachial plexus blockade: a randomized clinical trial. Reg Anesth Pain Med. 2015;40:133-8.

\section{Tables}

Table 1. Patient characteristics and surgical data. 


\begin{tabular}{lc}
\hline Age (years) & $61 \pm 9$ \\
Sex (male/female) & $10 / 9$ \\
Weight $(\mathrm{kg})$ & $65 \pm 10$ \\
Height $(\mathrm{cm})$ & $162 \pm 9$ \\
BMI $\left(\mathrm{kg} / \mathrm{m}^{2}\right)$ & $24.7 \pm 2.4$ \\
ASA physical status (I/II/III) & $6 / 11 / 2$ \\
Duration of surgery (min) & $120 \pm 29$ \\
Surgical procedure & 19 \\
\hline
\end{tabular}

Values are expressed as number or mean (SD). ASA $=$ American Society of Anesthesiologists, A/S, arthroscopic.

Table 2. Block-related outcomes.

\begin{tabular}{lc}
\hline Performance time (min) & $6.5(4.9-12.0)$ \\
Number of needle pass (n) & $4(3-6)$ \\
Patient discomfort (NRS 0 - 10) & $3(2-3)$ \\
Block duration (min) & $840 \pm 266$ \\
\hline
\end{tabular}

Values are expressed as mean (SD) or median (interquartile ranges). NRS = numerical rating scale. 
Table 3. Adverse events

\begin{tabular}{lcc}
\hline & PACU & $24 \mathrm{~h}$ \\
\hline Paresthesia (n) & 17 & 5 \\
Handgrip weakness (complete/partial/no) & $8 / 10 / 1$ & $0 / 1 / 18$ \\
& & \\
\hline Dyspnea (n) & 0 & 0 \\
\hline
\end{tabular}

Values are expressed as number.

Figures 


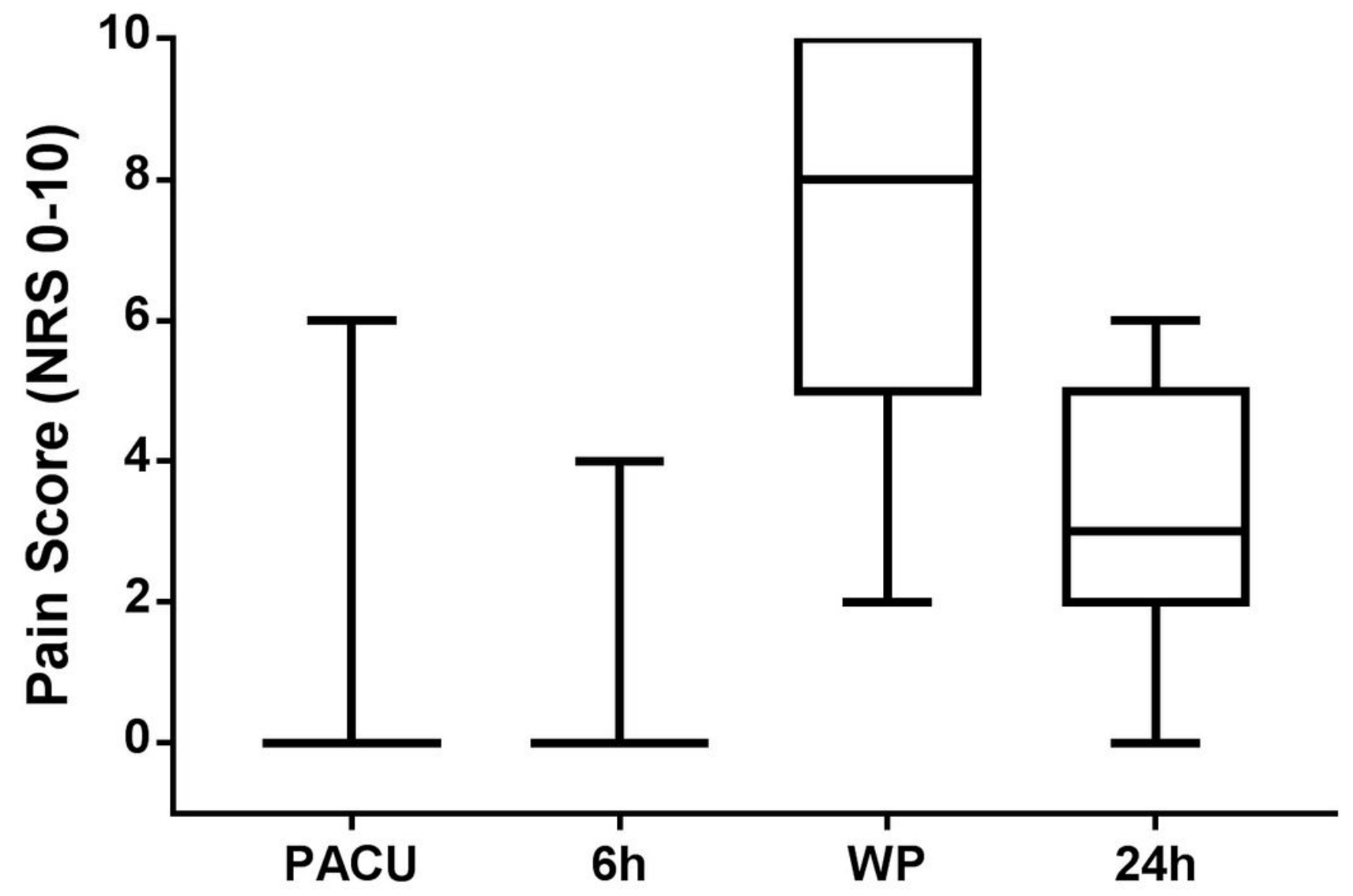

Figure 1

Postoperative pain scores at rest during the first 24 hours after surgery. NRS = numerical rating scale, PACU = post-anesthesia care unit, WP = worst pain. 